\title{
FASES DEL MODELO DIDÁCTICO-PROCESAL SEGUIDAS EN LA CONSTRUCCIÓN DE SIMULACIONES EN LA ASIGNATURA DE FÍSICA MÉDICA PARA EL CONTEXTO DE ENSEÑANZA- APRENDIZAJE VIRTUAL
}

\author{
(PHASES OF THE DIDACTIC-PROCEDURAL MODEL FOLLOWED IN THE CONSTRUCTION \\ OF SIMULATIONS IN THE COURSE OF MEDICAL PHYSICS FOR THE VIRTUAL TEACHING- \\ LEARNING CONTEXT)
}

\author{
Ana B. Sánchez García \\ Francisco-Javier Cabrero Fraile \\ José-Miguel Sánchez Llorente \\ Universidad de Salamanca (España)
}

\section{RESUMEN}

Presentamos un análisis teórico-aplicado de las fases del modelo pedagógico seguidas en la construcción de simulaciones en el ámbito disciplinar de la Física Médica. Analizamos las aportaciones del modelo a la adquisición del conocimiento procesal en el campo de la educación médica, y por qué los simuladores son útiles en la enseñanza de procesos. Así mismo, informamos de una experiencia llevada a cabo mediante la construcción de una simulación interactiva sobre el fenómeno de resonancia magnética, a través de los pasos de dicho modelo. Esta simulación fue utilizada como recurso de enseñanza en una plataforma de docencia virtual.

Hacemos hincapié en contribuciones interdisciplinares de la Psicología Cognitiva, la Informática, las Ciencias de la Educación, la Física y la Medicina. Todas ellas, proporcionan información para el análisis de los elementos relevantes en la adquisición de las habilidades necesarias para la resolución de problemas en física médica a través de simuladores.

Palabras clave: educación médica, física médica, enseñanza virtual, simulación computacional.

\begin{abstract}
This research paper is a theoretical and applied analysis of the phases of the pedagogical model followed in the construction of simulations in the field of Medical Physics. There is also an analysis of how the model contributes to the acquisition of procedural knowledge in medical education and how simulators are useful for the teaching of processes.
\end{abstract}


By following the phases of this model in a particular case, some specific information has been obtained about an interactive simulation which was designed for the purpose of investigating the magnetic resonance phenomenon. This simulation was used as a teaching resource on a virtual education platform. It is important to note the interdisciplinary contributions of Cognitive Psychology, Information Technology, Education Sciences, Physics and Medicine. All of these fields contributed to the analysis of the way in which the acquisition of problem solving skills required in Medical Physics occurs through the use of simulations.

Keywords: medical education, medical physics, virtual education, computer simulation computational.

De la fusión entre los postulados teóricos de la ciencia cognitiva y la tecnología de la información, emergen modelos de aplicación al campo educativo. Como resultado se ha incrementado el número de sistemas informatizados aplicados al aprendizaje de conceptos y habilidades. En la actualidad esta tecnología, en todos los campos y especialmente en medicina, se ha incorporado en las aulas al objeto de mediar en los procesos de enseñanza-aprendizaje. Autores como Anderson (1983, 1990), Newell (1990), VanLehn, Ohlsson y Nason (1994), Munro, Surmon y Pizzini (2006), se han centrado en describir el aprendizaje del conocimiento de tipo procedimental. Los resultados han revertido en un diseño instruccional que incorpora la tecnología de la simulación para representar los procesos que ha de llevar a cabo un estudiante al objeto de asegurar la consecución de una habilidad propuesta.

Con respecto al tema que nos ocupa, y antes de detenernos en los modelos de aprendizaje en el campo de la Física Médica, vamos a realizar una breve introducción a los sistemas tutores inteligentes centrándonos en los que abordan el conocimiento procesal, básico para la adquisición de conceptos a través de simulaciones.

Los simuladores son sistemas inteligentes que podemos incluir dentro de los llamados ITS's (Intelligente Tutoring Systems). El término inteligencia, relacionado con la capacidad de adaptación del sistema a los diferentes niveles de aprendizaje que manifiesta el estudiante, surge en la medida en que el sistema define el conocimiento de domino, incorpora un proceso tutorial y tiene la capacidad de representar el conocimiento que posee el aprendiz. Se basan pues, en un proceso interactivo y dinámico entre tutor virtual y alumno expuesto a una secuencia de aprendizaje previamente definida. Brusilovskiy (1994) describe su estructura compuesta por tres componentes principales: módulo de conocimiento experto, módulo del modelo estudiante y módulo tutor, tal como podemos apreciar en la figura 1. 


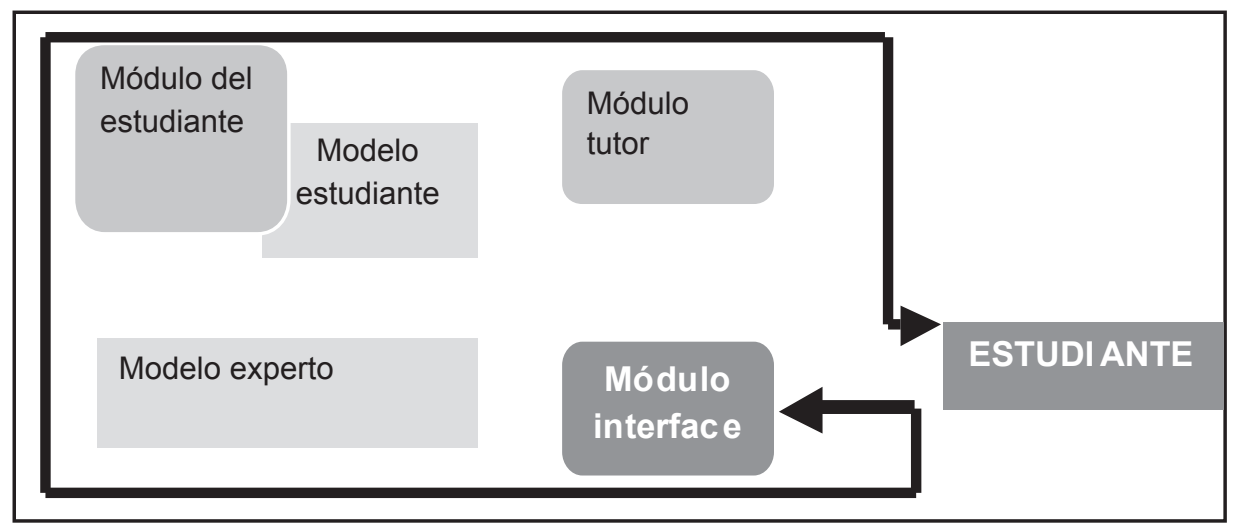

Figura 1. Estructura de un ITS `s adaptada de Brusilovskiy (1994)

El módulo experto: comprende el conocimiento que tiene que aprender el estudiante y está relacionado con el módulo tutor que guiará el proceso de enseñanza.

El modelo de estudiante: es una base de datos que contiene información del estudiante y permite la adaptación del sistema a los niveles de competencia que presenta el estudiante en relación a determinado contenido curricular. Su objetivo es desarrollar estos contenidos seleccionando el nivel óptimo para su enseñanza y aprendizaje, y facilitando el feedback adecuado a cada estudiante.

El módulo tutor: selecciona los procesos de enseñanza relacionados con el currículum y los objetivos previstos para la consecución de determinado dominio. Es un gestor pedagógico, que trabaja en función de la situación específica de aprendizaje y el comportamiento del alumno ante dicho material de aprendizaje, detectado a través de la interfaz.

La interfaz: es el entorno de comunicación y aprendizaje entre el sistema y el estudiante.

Estos sistemas representan los tipos de conocimiento como modelos de conocimiento procesal o declarativo. Por tanto, son prototipos aptos para la enseñanza de procesos, porque muestran el conocimiento a través de una red de procesos y subprocesos, en base a un conjunto de reglas o acciones relacionados con ellos. El objetivo más inmediato de los mismos es describir la secuencia de acciones, estrategias o pasos a seguir para completar el proceso a aplicar en otro contexto. Así pues, los estudiantes primero desarrollan los esquemas cognitivos necesarios para 
aprender dicha habilidad en el contexto de demostraciones interactivas simuladas, después ejecutan el proceso en otro contexto a través de un mecanismo de aprendizaje asociativo (Munro, Surmon y Pizzini, 2006). Según Feldman (1995), la simulación es un conjunto de procesos que existen. Sin embargo, en Física Médica, lo que diferencia a la simulación de otras técnicas es la naturaleza dinámica e interactiva que la caracteriza. Estas dos últimas peculiaridades son las que configuran la simulación como una metodología potencialmente pedagógica para la enseñanza de la física. De acuerdo con Shirts (1975), una simulación representa un conjunto de reglas que definen un modelo que refleja el mundo real. Un ejemplo aplicado a la Física Médica podrían ser las leyes que definen cómo los neutrones y átomos se mueven e interaccionan los unos con los otros en el caso de la fisión nuclear. Para ello podemos utilizar un reactor nuclear que simule la posibilidad de contener reacciones y el cálculo de la energía y potencia.

El aprendizaje de estos procesos en Física Médica a través de simulaciones comprende esencialmente tres fases generales que describen Fitts y Posner (1967). Estas fases coinciden con un primer momento de aprendizaje que se denomina cognitivo, y un segundo estado de aprendizaje asociativo, que ocurre durante la práctica de los procesos. Es decir, primero los estudiantes aprenden los elementos cognitivos del contenido en el contexto de demostraciones interactivas y ejemplificaciones, y posteriormente establecen un tipo de aprendizaje asociativo relacionado con la práctica. Finalmente, los estudiantes desarrollan la habilidad de ejecución del proceso de manera que llega a ser automática.

De acuerdo con Munro, Surmon y Pizzini (2006), para que se produzca este tipo de aprendizaje procedimental, el proceso de enseñanza ha de contemplar los siguientes componentes cognitivos: procesos de aprendizaje que comprenden una secuencia de acciones, aprendizaje de las condiciones bajo las cuales han de desarrollarse las acciones, y por último, los elementos que potencien los procesos de transferencia de esas acciones y condiciones a otros contextos.

Teniendo como referente estos componentes, y siguiendo a Clark (2004), la enseñanza del conocimiento procesal ha de incluir los elementos que mostramos en la figura 2: 


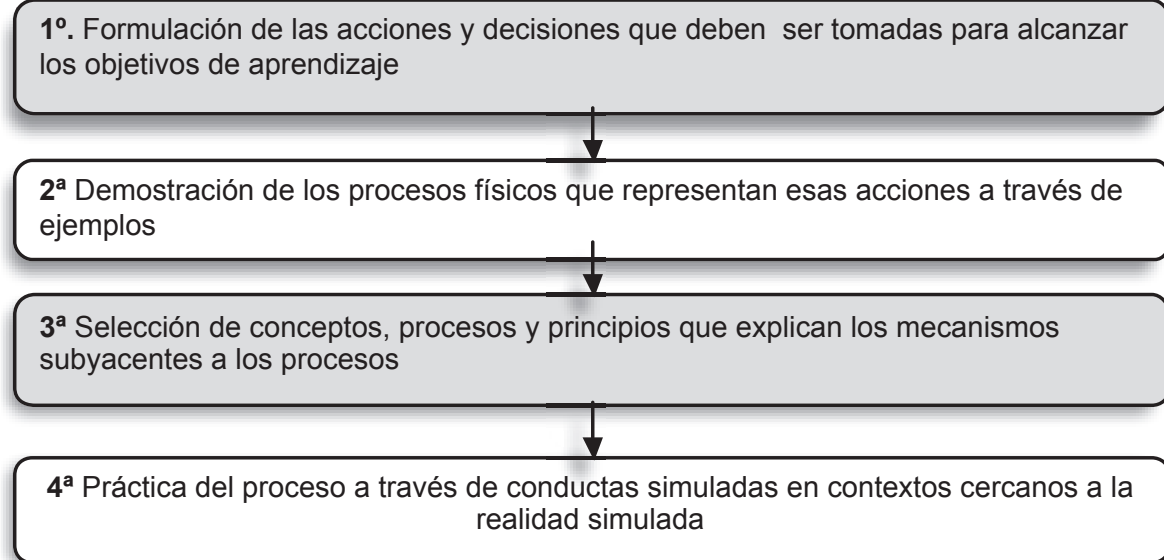

Figura 2. Fases del modelo didáctico en la enseñanza del conocimiento procesal mediante simuladores

Los componentes descritos en la figura 2 pueden ser representados a través de simuladores, ya que esta tecnología permite mostrar el aprendizaje de los contenidos como una secuencia de acciones, y además porque potencia los procesos transferenciales de esas acciones a otros contextos.

La información que describimos en este trabajo está fundamentada en la afirmación que hemos defendido en el párrafo anterior. No obstante, antes de presentar un modelo de simulador que hemos desarrollado, y dada la importancia dela transferencia para la adquisición de conceptos establecidos en el currículo de nuestra disciplina, haremos un breve inciso para describir los procesos transferenciales del conocimiento propio de la Física Médica. Es decir, cómo aprenden los alumnos de Medicina los procesos propios de la Física Médica y cómo los transfieren a otros contextos.

\section{PROCESOS TRANSFERENCIALES EN EL APRENDIZAJE DE CONCEPTOS DE FÍSICA MÉDICA}

Los estudios de transferencia han estado estrechamente relacionados con la teoría del asociacionismo. Con ello, el punto de vista adoptado era que los procesos de transferencia se caracterizaban por la aplicación y desarrollo de comportamientos semejantes desde un aprendizaje inicial de una tarea dada a una nueva tarea. Desde esta perspectiva, a un alumno el aprendizaje de $X$ le sirve para aprender $Y$; si $Y$ contiene elementos idénticos a $X$. Thorndike (1924) constituye la referencia básica 
de esta perspectiva específica en los procesos de transferencia. Otra perspectiva influyente en el estudio de los procesos transferenciales es la aportada por la Psicología de la Gestalt. Desde esta corriente conceptual, la transferencia eficiente ocurre cuando la misma estrategia general que fue aprendida en la resolución del problema $X$ es requerida para la solución del problema $Y$. A diferencia de Thorndike, para estos autores la transferencia puede transportar estrategias de carácter general, y no solo comportamientos y destrezas particulares. Enfatiza pues, en la existencia de dos tipos de transferencia si atendemos al contenido, la transmisión de estrategias generales y la transmisión de conocimiento específico. Las implicaciones educativas son inmediatas, puesto que la transferencia en la resolución de problemas es un objetivo en sí mismo, y por tanto, el método significativo es más eficientemente útil que los métodos meramente repetitivos, memorísticos o imitativos.

Otro punto de vista es el aportado en el ámbito del desarrollo de las teorías sobre el procesamiento de la información. En este contexto, la transferencia debe ser entendida como activación del conocimiento en las redes de memoria. Cuanto mayor número de vínculos y asociaciones existan entre las unidades de información, más probable será la activación de un conocimiento y, con ello, su aplicación transferida a otro contexto.

Las ideas anteriores dirigen nuestra atención al proceso de transferencia del conocimiento procedimental en Física Médica y su relación con el trabajo con simulaciones. Las características del aprendizaje de procesos en esta disciplina convierten la transferencia de conocimiento en un aspecto esencial para delimitar la naturaleza de la comprensión y aplicación de los aprendizajes a otros contextos. Desde la perspectiva que defendemos, el éxito del proceso transferencial viene determinado por la capacidad del sujeto para desarrollar las diferentes fases de un determinado proceso resolutivo (Sánchez y López, 2011). Es decir, la transferencia para la resolución de un nuevo problema en Física Médica está relacionada con el aprendizaje previo de estrategias generales, procedimientos específicos y técnicas particulares, que son en última instancia quienes posibilitan el desarrollo de las funciones metacognitivas de control, selección y tutorización del proceso resolutivo y transferencial. En consecuencia, el transfer metacognitivo depende, esencialmente, de destrezas cognitivas de carácter general que, evidentemente, se adquieren y fortalecen a través de ejercicios mentales y actividades desarrolladas sobre un conjunto y variedad de problemas que podemos presentar con la tecnología de las simulaciones. 
Por tanto, la transferencia de procesos se articula en base a un proceso positivo de transmisión de una secuencia de pasos que configuran el procedimiento desde una situación o estado inicial a otra situación final. De esta manera, influye la primera en la segunda de forma significativa, porque a mayor comprensión de la situación inicial mejor se transfieren los componentes a las situaciones finales relacionadas conceptualmente. Es decir, podríamos encontrar que el ejercicio para la mejora de un factor puede mostrar determinada influencia para el desarrollo de otros; influencia que podríamos denominar transferencia positiva. Por ejemplo, una metodología basada en técnicas de simulación que permita el entrenamiento en la adquisición de competencias tales como conocimiento del fundamento de la fisión de un núcleo atómico tras bombardeo con neutrones, favorece o refuerza la expansión del modelo a otros núcleos u otros fenómenos físicos de mecánica nuclear. Este tipo de transferencia se caracteriza por una economía en el aprendizaje de la tarea, que es debida al entrenamiento anterior en una tarea diferente, y estaría compuesta por el uso del conocimiento en circunstancias diferentes a las cuales fue adquirido. Es, pues, un sistema adaptativo que facilita la relación efectuada por la transferencia, y por tanto, el uso efectivo del procedimiento de fisión de un núcleo tras el bombardeo con neutrones.

\section{Resolución de problemas en Física Médica, las estrategias de transferencia y los simuladores}

Tanto la resolución de problemas en Física Médica, como la transferencia de aprendizaje que hemos definido anteriormente, constituyen dos núcleos relacionados. Efectivamente, en la investigación sobre la aplicación de la informática a la enseñanza de la Física, hemos de tomar como referencia modelos cibernéticos generales que simulen procesos de resolución de problemas y contextos transferenciales. El modelo de Ernesty Newell (1969) se basa en una estructura procesal que puede ser aplicada al desarrollo de simulaciones por ordenador que presentamos en la figura 3. Este modelo representa la versión más cercana al diseño del espacio de resolución de problemas mediante simulaciones en Física Médica. 


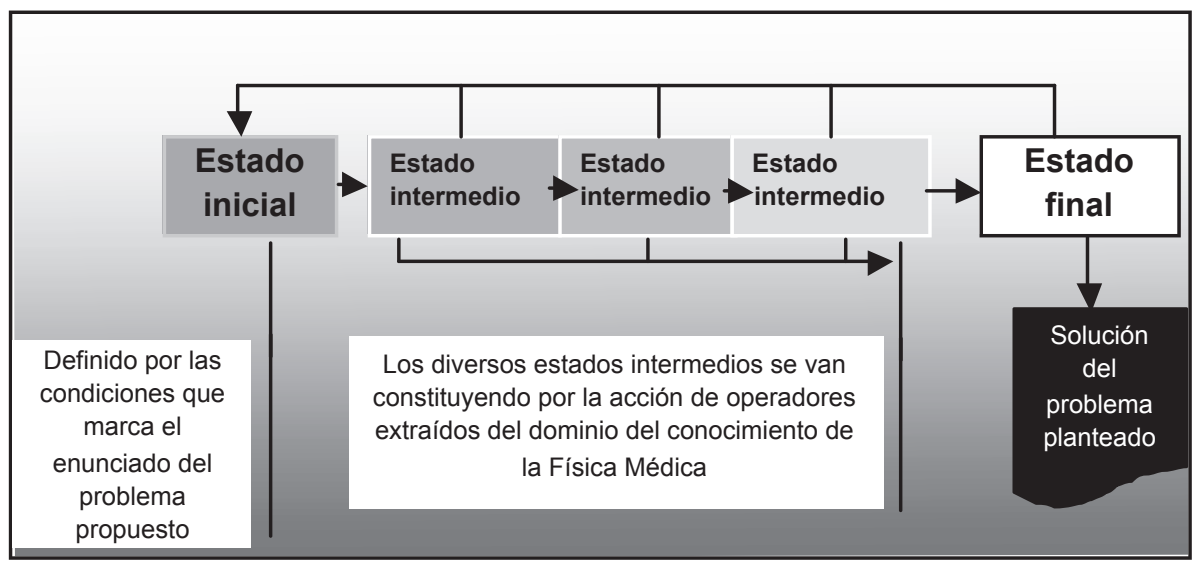

Figura 3. Estructura procesal de aplicación al desarrollo de simuladores en Física Médica. Adaptada de la concepción inicial de Simon en torno al proceso de resolución de problemas (1979)

Sobre la concepción dinámica del concepto de resolución de problemas presentada en la figura 3, Simon (1979) estableció la denominada heurística de análisis de medios y fines que fue esencial para el desarrollo histórico de las primeras aplicaciones informáticas aplicadas al campo de resolución de problemas. El proceso pormenorizado de la metodología generada por el análisis de medios y fines se configura sobre una secuencia de fases de producción que hemos aplicado al modelo pedagógico para la construcción de nuestras simulaciones. Dichas fases de producción se configuran en torno a la secuencia ordenada de los procesos que mostramos en la figura 4.

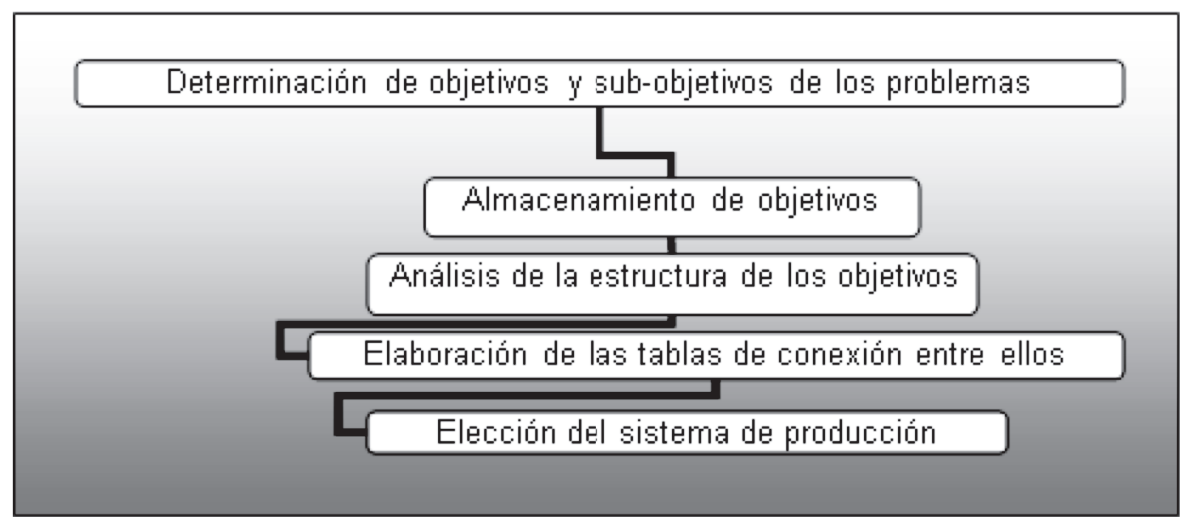

Figura 4. Procesos seguidos en la producción del modelo pedagógico utilizado para la construcción de nuestras simulaciones 
El proceso de resolución de problemas en Física pone en juego distintos sistemas de representación, entre ellos el heurístico. Si analizamos la naturaleza de las funciones metacognitivas asociadas al sistema heurístico, observamos que están relacionadas con procesos de control, elección, autorización, monitorización, y por último, evaluación. Todas ellas desarrolladas al objeto de modificar, en su caso, la estructura dinámica del sistema de producción asociado a la resolución del problema (López, 1999). Dicha modificación de la estructura del sistema de producción es llevada a cabo a través del ejercicio de la retroalimentación del propio proceso, y se contextualiza en el marco de una interacción entre el problema, sus condiciones y quien lo resuelve.

Por todo ello, podemos afirmar que el proceso de resolución de problemas en Física Médica para poder ser simulado requiere de un soporte que posea capacidad para almacenar redes proposicionales y sistemas de producción reticulares en su estructura, una estructura adaptativa y dinámica, interactividad, capacidad de control y regulación del sistema. Las características descritas son las que hemos intentado desarrollar en nuestras simulaciones, y por ello, como soporte constituyen un medio instrumental idóneo para emular procesos cognitivos y metacognitivos que operan en las tareas que proponemos. Por tanto, contribuyen a potenciar los procesos transferenciales en la disciplina en la cual trabajamos.

\section{DEFINICIÓN Y CARACTERÍSTICAS DEL PROCESO INSTRUCCIONAL EN LA ASIGNATURA DE FÍSICA MÉDICA IMPLEMENTADA EN UN ENTORNO VIRTUAL EN EL QUE SE INTRODUCEN LOS SIMULADORES}

De acuerdo con Tabakov (2008), la Física Médica se encuentra entre las primeras disciplinas profesionales que desarrollaron y aplicaron técnicas de e-Learning en el campo de las Ciencias de la Salud, como por ejemplo, la aplicación de simulaciones. Para el autor, existen una serie de elementos claves en la utilización del e-Learning en Física Médica. Entre ellas destaca que permiten una rápida y fácil actualización de los materiales de aprendizaje y proporcionan una vía óptima para la resolución de problemas mediante la comprensión de fenómenos físicos complejos (Tavakov, 2005). En los últimos años hemos tomado como referencia estas potencialidades en la utilización de metodologías de aprendizaje con apoyo del e-Learning. Por ello, en la construcción del modelo pedagógico que utilizamos en la plataforma virtual, nos apoyamos entre otros recursos en el uso de simulaciones que mostramos en este trabajo. De este modo, para el trabajo con las simulaciones que presentamos, optamos por un sistema de gestión de cursos (LMS) denominado FISIMED que incluía información sobre la materia, presentaciones PowerPoint, Software 
Multimedia, galerías de imágenes y vídeos, test de autoevaluación y simulaciones, entre otros. En la actualidad, nuestro esfuerzo se dirige especialmente al diseño, desarrollo e implementación de animaciones y simulaciones computacionales, aunque hemos migrado los contenidos de FISIMED a la plataforma de enseñanza online de la Universidad de Salamanca (Studium).

Para reforzar las afirmaciones realizadas en el marco teórico, nos referiremos en este apartado a una simulación, desarrollada como apoyo a la docencia de las asignaturas Radiología General, Medicina Física y Física Aplicada, de primer curso de la licenciatura de Odontología, y Física Médica de primer curso de la licenciatura de Medicina, que servirá para concretar lo expuesto hasta ahora y que intenta "hacer visible” al alumno el fenómeno de resonancia frente al de relajación, claves para la comprensión de los fundamentos físicos de la resonancia magnética.

Uno de los avances tecnológicos más importantes alcanzados en el campo de la radiología digital ha sido la consecución de imágenes basadas en la resonancia magnética. La resonancia magnética emplea campos magnéticos y radiación no ionizante de radiofrecuencia del espectro electromagnético en la obtención de dichas imágenes. Los fenómenos de resonancia y relajación se representan mediante vectores de magnetización, longitudinal (eje z) y transversal (plano x-y), de manera que:

"el vector de magnetización $M$ describe durante la relajación una espiral ascendente, una superficie en forma de pabellón de trompeta que es el resultado de los fenómenos de relajación longitudinal y transversal que ocurren simultáneamente. Sin embargo, durante la eXCITACIÓN, EL VECTOR DE MAGNETIZACIÓN DESCRIBE UNA ESPIRAL FORMANDO UNA SUPERFICIE EN FORTa de esfera debido a la reducción progresiva de su componente longitudinal y a la aparición de un componente transversal" (figura 5) (Cabrero, 2004, p. 177).

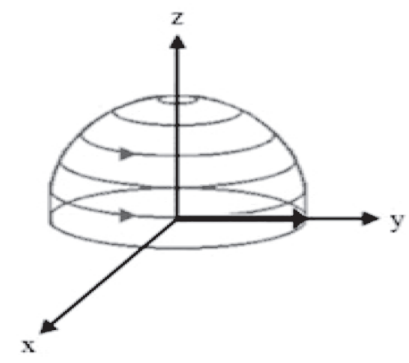

(a)

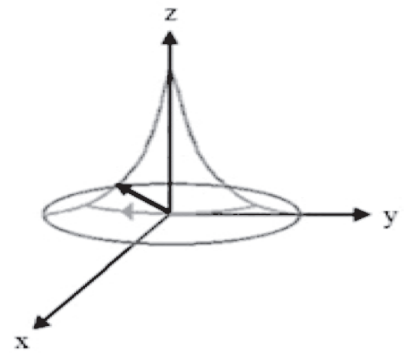

(b)

Figura 5. Excitación (resonancia) frente a relajación. (Tomado de Cabrero, 2004) 
La aplicación se realizó con el programa “Adobe Shockwave”. En los últimos años las simulaciones han experimentado un gran avance con la aparición de software destinado exclusivamente a estos propósitos. Por otro lado, otros programas como el Macromedia Flash, no destinados directamente a realizar simulaciones de forma exclusiva, se han usado al estar disponibles fácilmente y poder ser utilizados con un grado de formación medio, no requiriéndose grandes habilidades y obteniendo un resultado aceptable.

Flash incorpora el "Object Oriented" action script para la animación de formas y transición de escenas. En la simulación que se presenta se ha utilizado esta funcionalidad combinada con la posibilidad de ejecución matemática del programa Mathematica (Wolfram Research: sistema de álgebra computacional) a partir de las ecuaciones preestablecidas. La figura 6 muestra una pantalla en la que se observa la formación de la superficie en forma de esfera que describe el vector de magnetización durante la excitación (Cabrero, 2010).

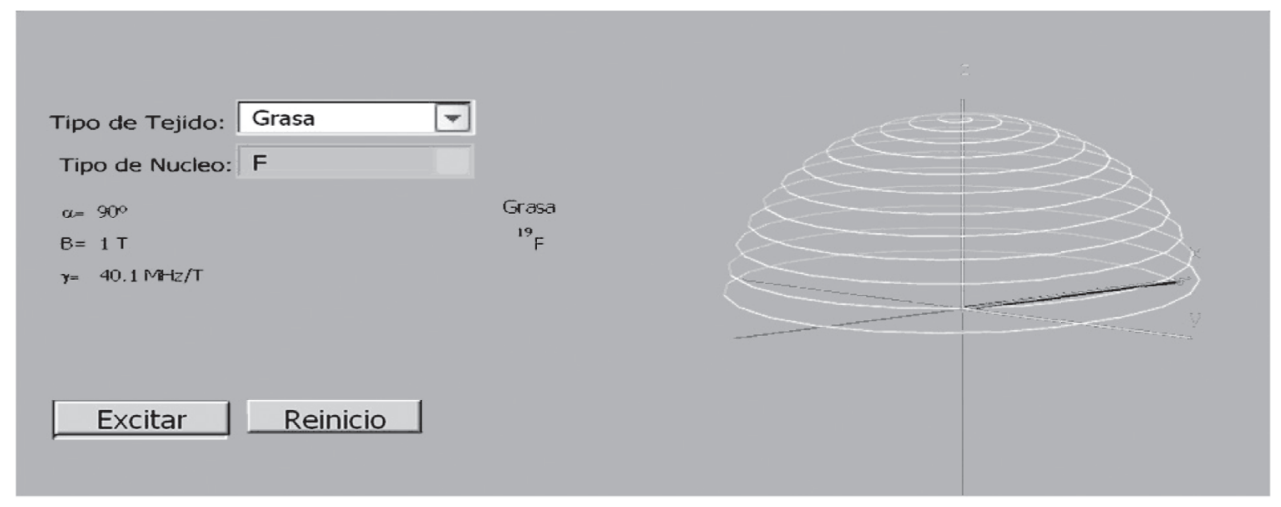

Figura 6. Simulación interactiva (fenómeno de resonancia magnética)

La arquitectura de la simulación sigue la estructura básica de cualquier sistema de animación que busque tutorización. Está compuesta por tres componentes principales analizados en apartados anteriores: módulo de conocimiento experto, módulo del modelo estudiante, y módulo tutor. En la siguiente figura se establece dicho modelado. 


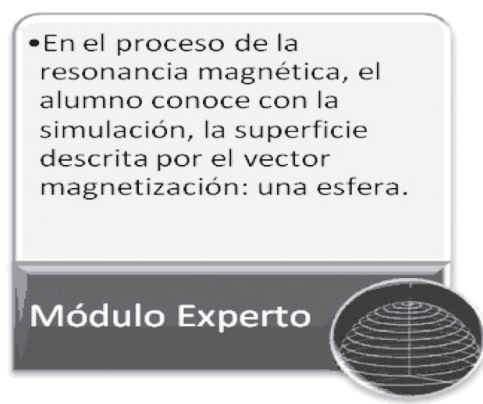

- Basado en Fisimed, herramienta multicanal que registra información del estudiante y permite la adaptación del sistema a los niveles de competencia que presente en relación a la materia de Física Médica

\section{Módulo Modelo Estudiante}

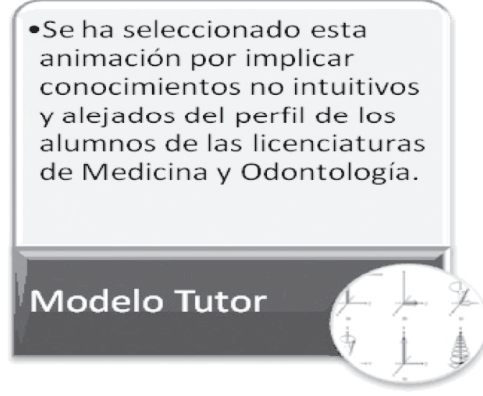

- La animación de Spin se encuentra disponible en Fisimed en su canal ligero: vía internet y pesado: software codificado en $\mathrm{C} \# \mathrm{y}$ . Net y disponible en ordenadores de la Facultad de Medicina

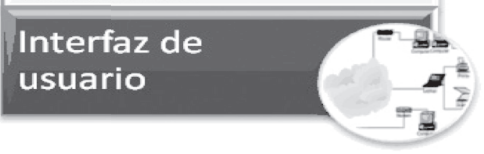

Figura 7. Estructura de la simulación interactiva (fenómeno de resonancia magnética).

Teniendo en cuenta el marco teórico que hemos expuesto en anteriores apartados, el proceso de desarrollo de esta simulación sigue el esquema de fases que exponemos a continuación. 


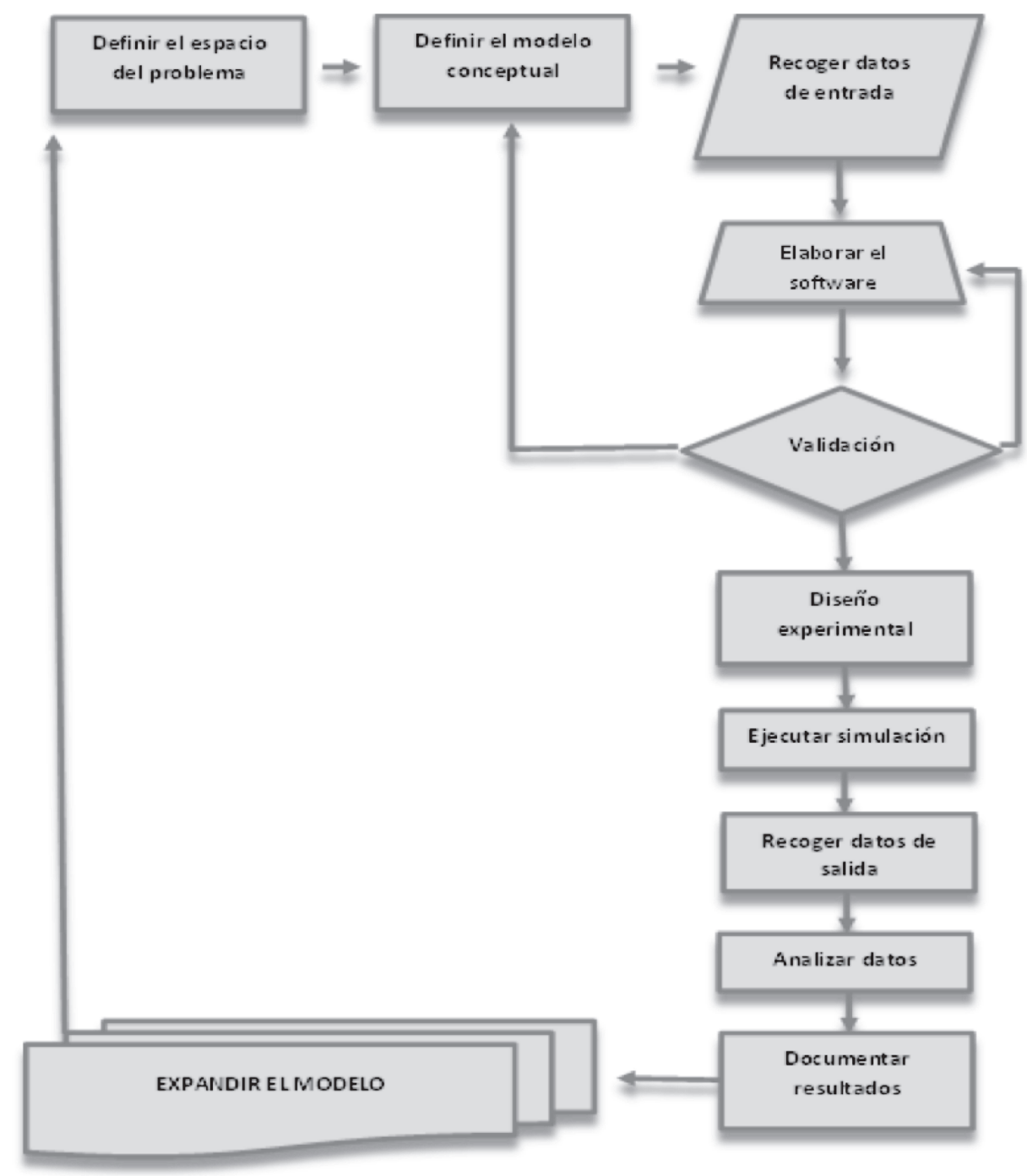

Figura 8. Fases del modelo pedagógico seguidas en la construcción de la simulación fenómeno de resonancia magnética

\section{DISCUSIÓN Y CONCLUSIONES}

A lo largo de este trabajo hemos desarrollado el marco teórico que fundamenta el porqué de la eficacia de las simulaciones. Algunas respuestas a la cuestión están determinadas por las características propias de la enseñanza a través de simuladores 
caracterizados por su naturaleza dinámica e interactiva. Estas dos últimas particularidades son las que configuran la simulación, como una metodología potencialmente pedagógica para la enseñanza de la Física, y también de otras disciplinas que requieren del aprendizaje de procedimientos.

Del mismo modo, hemos hecho referencia a los procesos transferenciales. En este sentido, las simulaciones como soporte, constituyen un medio instrumental idóneo y potencian la transferencia del conocimiento a otros contextos, porque permiten la recreación virtual de múltiples metáforas estimuladoras del pensamiento analógico.

Por otra parte, la enseñanza del conocimiento procedimental requiere de metodologías didácticas específicas como son la demostración, ejecución de los pasos que componen el procedimiento, y la supervisión de la práctica (Munro, Surmon y Pizzini, 2006). Estas metodologías pueden ser desarrolladas óptimamente en contextos de enseñanza virtual gracias a los simuladores.

En el plano de lo estrictamente pedagógico, para que la construcción de simulaciones sea eficaz hemos de contemplar determinados componentes que hemos incorporado al modelo didáctico-procesal. No obstante, el valor educativo de la utilización de simuladores va más allá de su uso en nuestro campo disciplinar, y por tanto, este modelo didáctico puede ser exportado a otras disciplinas que requieran del aprendizaje de procesos. En este sentido, de acuerdo con Shoikova y Tzanova (1999), una razón para usar simulaciones en contextos educativos es que ayudan al aprendizaje activo y maximizan el control del alumno sobre su proceso de estudio. Bajo esta premisa, la mayor parte de los estudios relacionados con el tema informan positivamente sobre la utilización de simulaciones informáticas en distintas disciplinas educativas (Monsky et al., 2002; Weller, 2004), puesto que permite a los alumnos disponer de una ayuda para la comprensión de conceptos que no pueden ser "visualizados" en el aula o en el laboratorio. De manera específica, también contamos con algunos estudios que avalan la utilización de los mismos en contextos de e-Learning. En concreto, de acuerdo con los resultados obtenidos por Camerón (2003), las simulaciones en entornos virtuales permiten la aplicación de conocimiento a la solución de problemas, potencian la comprensión de conceptos abstractos, mejoran los procesos transferenciales y, por último, aumentan la motivación del estudiante, porque mejora su percepción sobre el control de su propio aprendizaje.

Así pues, en la docencia virtual la aportación más importante de estos recursos es la ayuda que ofrecen a los estudiantes en la adquisición de un tipo conocimiento 
basado en la práctica y centrado en el alumno. Si además tenemos en cuenta que la mayor parte de los cursos virtuales se convierten en repositorios de objetos de aprendizaje pasivos basados en texto (documentos Word, ppt., pdf...), la relevancia de estos desarrollos en la enseñanza virtual es indiscutible.

De este modo, un entorno virtual que contenga simulaciones se configura como entorno potenciador de aprendizajes significativos en cualquier disciplina, porque además de ofrecer la experiencia de aprendizaje a través de la simulación, ofrece otros recursos como tutoriales, información complementaria, posibilidad de establecer comunicación tanto con el profesor como con el resto de estudiantes, etc.

En resumen, los entornos virtuales que implementan simulaciones pueden ser considerados como entornos puros de desarrollo de la cognición situada descrita por autores como Rogoff (1993), Lave (1997), Wenger (2001), porque en ellos se prioriza la actividad y un contexto de aprendizaje que fomenta el aprender haciendo. Es decir, los simuladores privilegian el saber cómo, y son complementados por otras herramientas que privilegian el saber qué. Un ejemplo de ello es el "MIT OPEN CURSE WARE" del Instituto Tecnológico de Massachussets (http://ocw.mit.edu/ index.htm), plataforma virtual en la que se combinan todo tipo de herramientas mediadoras en el proceso de enseñanza-aprendizaje y se potencia la utilización de simuladores en la adquisición del conocimiento procedimental.

Finalmente, en la implementación de simuladores en la plataforma virtual utilizada en nuestra disciplina hemos hecho converger a distintos sectores profesionales (médicos, odontólogos, físicos, pedagogos, psicólogos, informáticos,...) en un proceso de investigación interdisciplinar que aporta un valor añadido a los trabajos, ya que puede estimular la innovación e investigación, y contribuir a la mejora de la calidad de la enseñanza. 


\section{REFERENCIASBIBLIOGRÁFICAS}

Anderson, J. (1983). The architecture of cognition. Cambridge, Massachusetts, EE.UU.: Harvard University Press.

Anderson, J. (1990). The Adaptive Character of Thought. Lawrence Erlbaum.

Brusilovskiy, P. (1994). The Construction and Application of Student Models in Intelligent Tutoring Systems. Journal of Computer and Systems Sciences International, 32 (1), (70-89).

Cabrero, F. J. (2004). Imagen radiológica. Principios físicos e instrumentación. Barcelona: Masson, S. A.

Cabrero, F. J.; Sánchez, J. M.; Sánchez, A. B.; Borrajo, J.; Rodríguez, M. J.; Cabrero, M.; Juanes, J. A. (2010). Simulaciones computacionales en la enseñanza de la Física Médica. TESI, 11 (2), (46-74).

Cameron, B. H. (2003). The effectiveness of simulation in a hybrid and online net working course. Quarterly Review of Distance Education, 4, (51-55).

Clark, R. (2004). What Works in distance learning: instructional strategies. En: O'Neil, H. F. (Ed.). What works in distance learning: Guidelines. Greenwich, CT: Information Age Publishing, (25-40).

Ernst, G.; Newell, A. (1969). GPS: A Case Study in Generality and Problem Solving. New York: Academic Press.

Feldman, H. D. (1995). Computer-Based Simulation Games: A Viable Educational Technique for Entrepreneurship Classes? Simulation \& Gaming. An international Journal of Theory, Design and Research. Newbury Park, London, New Delhi: Sage Periodicals Press, 26 (3), (346-360).

Fitts, P. M.; Posner, M. I. (1967). Human Performance: Basic concepts in Psychology Series. Belmont CA: Boocks-Cole.

Lave, J. (1997). The culture of acquisition and the practice of understanding. En:
Kirshner, D.; Whitson, J. A. (Eds.). Situated cognition. Social, semiotic and psychological perspectives (17-35). Mahwah, NJ: Lawrence Erlbaum.

López, R. (1999). Desarrollos curriculares de la ciencia de computadores en la enseñanza elemental. Tesis doctoral no publicada. Universidad de Salamanca, Facultad de Educación, Salamanca. España.

Munro, A.; Surmon, D.; Pizzini, Q. (2006). Teaching Procedural Knowledge in Distance Learning Environments. En: O’ Neil, H. F.; Perez, R. S. (Eds). WebBased Learning. Theory, Research, and Practice. Mahwah, New Jersey: Lawrence Erlbaum Associates, (255279).

Newell, A. (1990). Unified Theories of Cognition. Cambridge, MA: Harvard.

Monsky, W. L.; Levine, D.; Mehta, T. S.; Kane, R. A.; Ziv, A.; Kennedy, B.; Nisnbaum, H. (2002). Using a sonographic simulator to assess residents before overnight call. $A J R$, 178, (35-39).

Rogoff, B. (1993). Aprendices del pensamiento. El desarrollo cognitivo en el contexto social. Barcelona: Paidós.

Sánchez, A. B.; López, R. (2011). La transferencia de aprendizaje algorítmico y el origen de los errores en la sustracción. Revista de Educación, 354,(429-445).

Shirts, R. G. (1975). Notes on defining "Simulation". En: Greenblat, C. S.; Duke, R. D. (Eds.). Gaming-simulation: Rationale, design and applications. A text with parallel readings for social scientists, educators, and community workers.Toronto: Wiley \& Sons.

Simon, H. A. (1979). Models of Thought. New Haven, CT: Yale University Press

Shoikova, E.; Tzanova, S. (1999). Innovaciones en la Educación Superior 
en Electrónica a través del Desarrollo de un Entorno de Aprendizaje Basado en Simulaciones y Conducido por Desarrollo de Proyectos. RIED. Revista Iberoamericana de Educación a Distancia, 2 (2), (125-135).

Tabakov, S. (2005). E-Learning in Medical Engineering and Physics. Journal of Medical Engineering and Physics, 27 (7), (543-547).

Tabakov, S. (2008). e-Learning development in medical physics and engineering. BiomedImaging Interv J., 4 (1):e27, (1-5). [En línea] Disponible en: http:// www.biij.org/2008/1/E27 (consulta 2012, 15 de marzo).
Thorndike, E. L. (1924). Mental discipline in high school studies. Journal of Educational Psychology, 15, (1-22).

Vanlehn, K.; Ohlsson, S.; Nason, R. (1994). Applications of Simulated Students: An Exploration. Journal of Artificial Intelligence in Education, 2, (135-175).

Wenger, E. (2001). Comunidades de práctica. Aprendizaje, significado e identidad. Barcelona: Paidós.

Weller, J. (2004). Simulation in undergraduate medical education: bridging the gap between theory and practice. Med Educ, 38, (32-8).

\title{
PERFIL ACADÉMICO Y PROFESIONAL DE LOS AUTORES
}

Ana B. Sánchez García. Doctora en Educación, Máster en Servicios Sociales. Ayudante Doctor del Dpto. de Didáctica, Organización y Métodos de Investigación. Investigadora en diversos proyectos I+D+I. Ha desarrollado tareas de coordinación de cursos de Formación del Profesorado en el I.U.C.E. de la Universidad de Salamanca. Asesora de Investigación en el CITA. Ha desarrollado proyectos de evaluación educativa y tecnológica. Cuenta con publicaciones en el ámbito de la Didáctica aplicada al campo de las Matemáticas y las TIC, y educación.

E-mail: asg@usal.es

DIRECCIÓN DE LA AUTORA

\author{
Ana B. Sánchez García \\ Facultad de Educación, Universidad de \\ Salamanca \\ Ps/Canalejas 169, Edificio Cossio, \\ Salamanca. 37008, España
}

Francisco J. Cabrero Fraile. Doctor en Medicina y Cirugía. Profesor Titular del Departamento de Física, Ingeniería y Radiología Médica de la Facultad de Medicina de la Universidad de Salamanca. Ha desarrollado tareas de gestión formando parte del equipo del decanato como secretario. Investigador en diversos proyectos I+D+I. Sus líneas de investigación son la Imagen Radiológica, Innovación 
Docente en Radiología. Cuenta con numerosas publicaciones en el ámbito de la Radioterapia, Educación Médica y Medicina Radiológica.

E-mail: cabrero@usal.es

DIRECCIÓN DEL AUTOR

\author{
Francisco J. Cabrero Fraile \\ Facultad de Medicina, Universidad de \\ Salamanca \\ Calle de Alfonso X 'El Sabio', \\ 37007, Salamanca, España
}

José M. Sánchez Llorente. Doctor en Física. Profesor Asociado del Departamento de Física General y de la Atmósfera de la Facultad de Ciencias de la Universidad de Salamanca. En la actualidad es gerente del Parque Tecnológico de la Universidad de Salamanca. Investigador en diversos proyectos I+D+I. Sus líneas de investigación son la Imagen Radiológica, Innovación docente en Radiología y Física Médica. Cuenta con numerosas publicaciones en el ámbito de la Radioterapia, Educación Médica y Medicina Radiológica.

E-mail: jmsll@usal.es

DIRECCIÓN DEL AUTOR

José M. Sánchez Llorente

Facultad de Física, Edificio de Ciencias.

Plaza de los Caídos, s/n

37008, Salamanca, España

Fecha de recepción del artículo: 04/09/11

Fecha de aceptación del artículo: 13/12/11

\title{
Como citar este artículo:
}

Sánchez García, A. B.; Cabrero Fraile, F. J.; Sánchez Llorente, J. M. (2012). Fases del modelo didáctico-procesal seguidas en la construcción de simulaciones en la asignatura de Física Médica para el contexto de enseñanza-aprendizaje virtual. RIED. Revista Iberoamericana de Educación a Distancia, volumen 15, $\mathrm{n}^{0}$ 2, pp. 13-30. 